\title{
Towards Reliable and Lightweight Communication in Industrial Wireless Sensor Networks
}

\author{
Filip Barać*, Kan Yu ${ }^{\dagger}$, Mikael Gidlund ${ }^{\ddagger}$, Johan Åkerberg ${ }^{\ddagger}$ and Mats Björkman ${ }^{\dagger}$ \\ ${ }^{*}$ Mid Sweden University, Sweden \\ ${ }^{\dagger}$ Mälardalen University, Sweden \\ $\ddagger$ ABB AB, Corporate Research, Sweden
}

\begin{abstract}
In this paper we address the issues of timeliness and transmission reliability of existing industrial communication standards. We combine a Forward Error Correction coding scheme on the Medium Access Control layer with a lightweight routing protocol to form an IEEE 802.15.4-conformable solution, which can be implemented into already existing hardware without violating the standard. After laying the theoretical foundations, we conduct a performance evaluation of the proposed solution. The results show a substantial gain in reliability and reduced latency, compared to the uncoded transmissions, as well as common Wireless Sensor Network routing protocols.
\end{abstract}

\section{INTRODUCTION}

The research efforts to introduce Wireless Sensor Networks (WSN) into Process Automation (PA) have produced three major industrial communication standards: WirelessHART [1], ISA100.11a [2] and WIA-PA [3]. The solutions based on these specifications shall satisfy the stringent performance requirements of PA applications and collect the sensor data in a reliable and timely manner. Nevertheless, a number of field tests, such as [4], reveal the fact that high communication reliability and real time performance can hardly be achieved. Some downsides of the existing solutions are worth mentioning. Routing protocols encountered in existing industrial communication standards are overcomplicated. Path failures in a dynamic environment (such as industrial) are frequent, triggering tedious path recalculation processes, during which transmission of data traffic is put on hold. Control message overhead is a natural side effect in such an environment, and exchange of routing tables, beacons and other control messages consumes bandwidth; Error Control and Correction: industrial communication standards do not approach the problem of error control in a preemptive manner. Instead, packet retransmissions are employed, which may boost the data latency in the network and deliver outdated data to the sink. Consequently, rethinking of current designs deserves consideration.

Flooding is the most rudimentary routing technique and flooding-based algorithms are often seen as a way to simplify data dissemination in WSNs. Traditional flooding approaches suffer from broadcast storm and energy inefficiency problems, which were thoroughly studied in [5] and [6]. The floodingbased schemes presented in [7], [8], [9] and [10] are designed for broadcast traffic. However, the aim of this work is to simplify sensor data collection, rather than point-to-multipoint communication. Flooding-based routing schemes encountered in the literature require no routing tables, path discovery nor routing information exchange. Akkaya and Younis [11] address the broadcast storm problem by randomizing the selection of retransmitters, which is unacceptable in industrial settings. A random routing strategy for WSNs in [12] reduces energy consumption at the expense of higher latency, which is unacceptable in PA applications. In [13] a directed flooding scheme is proposed, but, despite significant energy savings, the transmission reliability remains equal to the one of the regular flooding scheme. Although there exist numerous works on Forward Error Correction (FEC) for WSNs, only [14] (and references therein) and [15] provide packet-level implementation details. Furthermore, to the best of our knowledge, no solution that blends lightweight routing with FEC coding for WSNs can be found in the literature.

In this paper we combine a lightweight flooding-based routing protocol with a IEEE 802.15.4-compliant FEC coding scheme to improve the reliability and simplify routing in Industrial Wireless Sensor Networks (IWSN). The proposed solution is fully IEEE 802.15.4-compliant and incorporable into existing hardware without any interventions from the manufacturers. The routing scheme is entirely free of routing tables or control message exchange and data is delivered to the sink via multiple paths. Since there is no path recalculation in case of link failures, the network unavailability due to path reestablishment is eliminated. A packet delivery deadline is imposed, discarding outdated packets at intermediate nodes and preserving bandwidth. The second component of the proposed solution is an IEEE 802.15.4-compliant FEC coding scheme on the Medium Access Control (MAC) layer. Any systematic channel code is applicable, and the scheme allows even nodes without coding/decoding capability to participate in the network. In this work we also provide the theoretical analysis of the performance, followed by simulation verification, with respect to the performance constraints. The results show a considerable improvement of end-to-end delay and reliability introduced by the proposed solution, compared to several common WSN protocols, using both coded and uncoded transmissions.

In the sequel, Section II presents the proposed solution. Section III is a description of simulation setup and scenarios. Simulation results are presented and discussed in Section IV. Conclusions are drawn and summarized in Section V. 


\section{Proposed FEC-COded Lightweight Routing SCHEME}

The two essential real-time performance parameters in IWSN are latency and reliability. The proposed solution consists of two components. On the Network layer, a lightweight routing protocol is used, complemented with a simple and flexible MAC layer FEC coding scheme which conforms to the IEEE 802.15.4 standard. Since the standard is concerned only with Physical and MAC layers of the protocol stack, the proposed solution can be smoothly incorporated into the equipment available on the market. After presenting the two components of the solution in detail, we conduct a theoretical analysis of Packet Delivery Ratio (PDR) and end-to-end delay.

\section{A. The lightweight and reliable transmission scheme}

Flooding is the most basic routing technique and, as such, it is a desirable option in lightweight routing protocol design. Its most notable drawback is a possible network congestion caused by its broadcasting nature, which may be avoided by proper definition of forwarding criteria. The advantages of using flooding-based routing are two-fold: this transmission scheme is simple enough to increase network availability, due to absence of frequent route recalculation, and it allows the possibility of having multiple transmission paths. However, having redundant routing paths requires additional timeslots in a Time Division Multiple Access (TDMA) network and causes an increase in average end-to-end delay in the network. In other words, there exists a trade-off between latency and reliability when flooding-based approaches are considered. Hence, in order to prevent network congestion, the number of routing paths between source and destination must be kept at a moderate level. In this work, we strive to circumvent this tradeoff i.e. to improve the reliability without further increasing the number of redundant paths (which is kept at two in this work). A lightweight flooding-based routing protocol with redundant paths is combined with FEC coding, which results in further increase of reliability without further increasing the number of redundant paths. A certain amount of trade-off still remains, though - FEC coding comes at the expense of coding overhead, which reduces the useful payload size. Below we present the two building blocks of the proposed solution.

1) A Lightweight routing scheme: The first component of the proposed solution is a lightweight routing protocol for IWSN defined in [16]. It is a lightweight location-based data dissemination scheme with mechanisms to handle outdated packets. While data is delivered via multiple transmission paths, no routing infrastructure is necessary and there is virtually no control message exchange. This is achieved by storing all data necessary for routing inside a special section of transmitted data packets. Since the underlying concept of the routing component is flooding, the acknowledgements and packet retransmission are omitted. In conjunction with adequate scheduling, this routing protocol guarantees an upper bound on the latency of delivered packets.

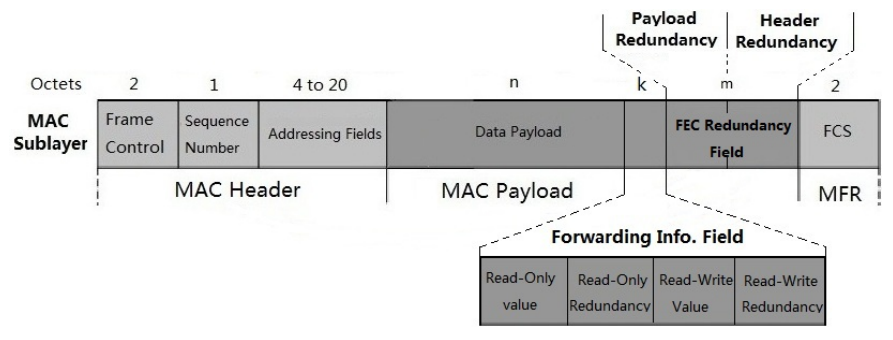

Fig. 1. Proposed Coded Data Frame format

2) Combining FEC with lightweight routing scheme: Forward Error Correction coding is a method to control symbol errors over unreliable channels, based on combining a redundancy of $n-k$ symbols with $k$ symbols of transmitted data. Direct FEC coding of entire packets at source has an obvious drawback - in this case, every forwarding node must decode the whole packet to extract the forwarding information and encode the whole packet again if forwarding criteria are fulfilled, which results in higher workload for all sensor nodes and higher energy consumption. Therefore, we combine the IEEE 802.15.4-compliant FEC coding scheme from [14] with the previously defined lightweight routing approach.

The values that need to be extracted from a packet for routing purposes are of two types. One is the read-only type, i.e. the values that are only read from the packet, but remain the same after each hop. The values of read-write type are updated after each hop. Since a systematic FEC code is applied, the redundancy can be parsed from codewords. We insert two additional fields into the coded packets, as shown in Figure 1: FEC Redundancy Field, consisting of MAC header and payload redundancy and Forwarding Information Field, that consists of four parts: read-only and read-write payloads, as well as their coding redundancies. From Figure 1 it can be noticed that the two newly introduced field types are encoded individually. Frame Check Sequence (FCS) is out of the FEC protection range and calculated at the last step, in order to be compatible with the IEEE 802.15.4 packet format.

The packet forwarding process in intermediate nodes is summarized in Algorithm 1. The correctness of a received packet can be checked by calculating FCS. If the packet is correct and contains the broadcast address, the forwarding condition check can proceed without any additional decoding activity. When all the forwarding conditions are fulfilled, before forwarding the packet, only read-write values need to be encoded again, rather than encoding the whole packet. Recalculation of FCS field is required as well. If errors are found in the received packet, the forwarding procedure will be slightly different. Before forwarding information value is read from the packet, it first needs to be decoded in order to recover the original data. All intermediate nodes should guarantee the correctness of packets, since transmission of corrupted packets unnecessarily consumes energy and bandwidth.

Therefore, an intermediate node shall drop the packet if decoding fails, i.e. the number of erroneous symbols exceeds the FEC code correction capability. Before forwarding a 


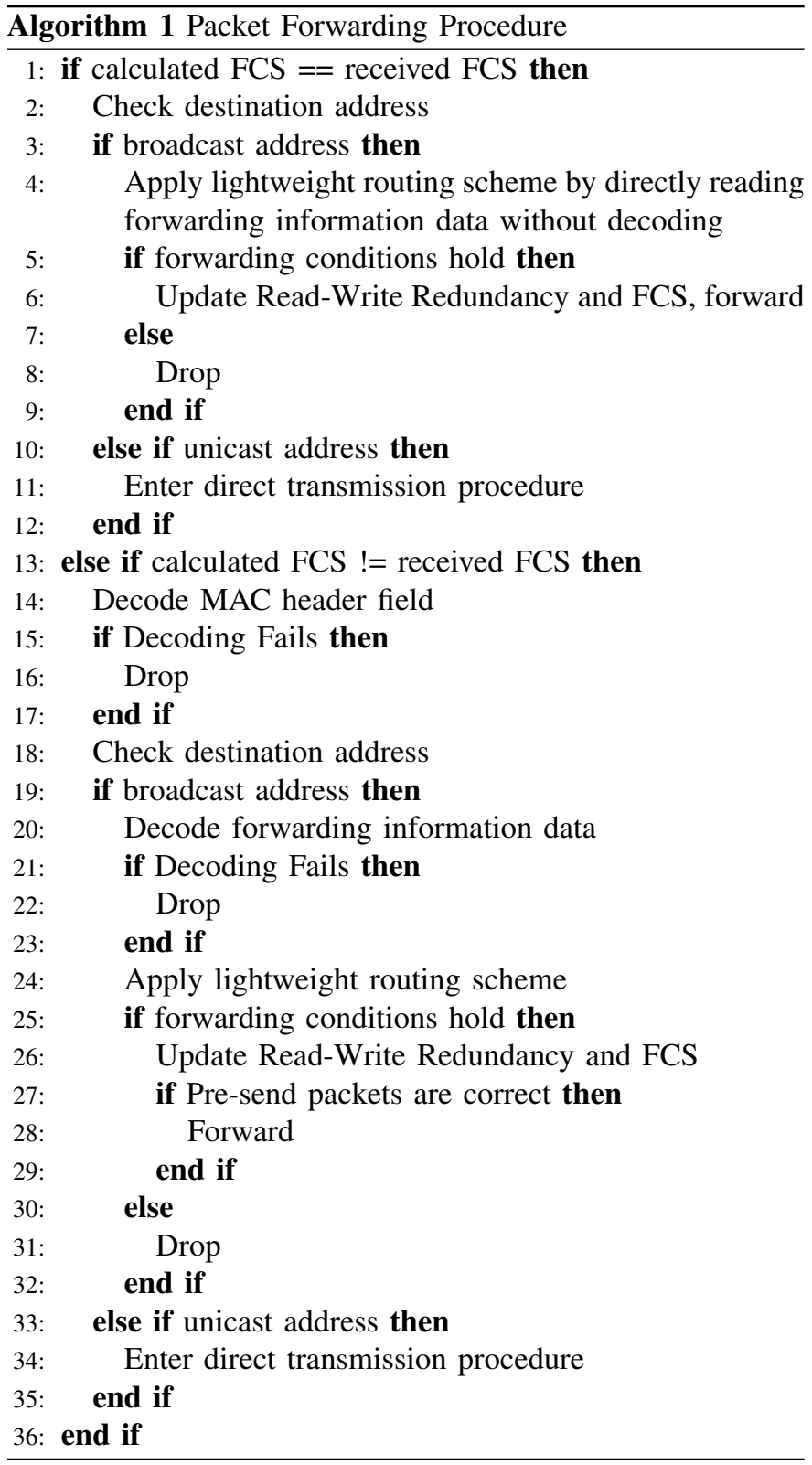

packet, an intermediate node shall recalculate the FCS value and compare it with the received FCS value. If they are different, the node can continue to decode other parts or drop the packet. It rarely happens that only received FCS value is corrupted, which can be detected by successful decoding of all other parts. In our approach, the number of FEC coding and decoding operations in the forwarding process is reduced to a minimum. When there is no error in the packet, decoding can be entirely avoided. Therefore, the proposed FEC-coded lightweight routing scheme will not significantly contribute to the workload of sensor nodes. Reference [14] evaluates the reduction in the number of decoding function calls of the FEC coding scheme used in this work.

The choice of FEC code is subject to the limited resources of sensor node hardware in terms of memory requirements and processing time. Moreover, the code rate of the chosen FEC code shall not be too low; otherwise more energy is spent to transmit the redundant data, leading to lower transmission efficiency. Another important aspect is the timing constraint in TDMA networks. When a node is scheduled for receiving or transmitting in two consecutive timeslots, it should be able to complete all necessary actions, including encoding or decoding the packet during the first timeslot, so it could be ready for following tasks in the next slot.

Taking WirelessHART for example, the TDMA timeslot duration is $T_{t}=10 \mathrm{~ms}$. Since the bit rate is $250 \mathrm{kbps}$, for a packet of 126 bytes and 6 bytes of preamble, the packet receiving time is:

$$
T_{\text {rec }}=\frac{132 \text { bytes } \times 8 \text { bits } / \text { byte }}{250 \mathrm{kbps}}=4.2 \mathrm{~ms}
$$

Besides the time required for listening and receiving a packet, the minimum time left for decoding within one slot is:

$$
T_{t}-\left(\text { TsR } x \text { Offset }+ \text { TsR } x \text { Wait }+T_{\text {rec }}\right)=2.256 \mathrm{~ms}
$$

Variables TsRxOffset and TsRxWait can be found in WirelessHART standard [1]. An investigation on a commercial sensor platform in [17] shows that $\mathrm{RS}(15,7)$ Reed-Solomon codes are simple and flexible enough for use in WSNs, with a decoding time of about $0.9 \mathrm{~ms}$. The block structure of ReedSolomon codes has an inherent benefit that it allows even partial decoding of the payload, which greatly reduces the amount of in-node processing.

\section{B. Theoretical Performance Analysis}

Real-time performance of the transmission scheme is evaluated by measuring: 1. Average end-to-end delay, defined as the packet travel time between the Application layers of the originating sensor node and the sink, and 2. Packet Delivery Ratio, i.e. the fraction of successfully delivered data packets to the sink within the deadline, relative to the total number of transmitted data packets. In this subsection, we provide a performance analysis of the proposed FEC-coded lightweight routing scheme, in terms of Packet Delivery Ratio (PDR) and end-to-end delay. We prove that the proposed scheme is able to increase the overall PDR and reduce the transmission latency.

1) Packet Delivery Ratio analysis: In the proposed lightweight routing scheme, packets are delivered to the sink via several possible routing paths. The number of paths depends on the IWSN topology, and if the topology allows the existence of multiple paths from every node to the sink, this will result in higher PDR and improved reliability.

Consider a packet having to travel $N$ hops from the source to the sink. $P_{e}^{(h o p)}(k)$ is the uncoded Packet Error Rate (PER) from the $k$ th hop to the $k+1$-st hop. Then PDR of this path $P_{d}^{(\text {path })}$ is calculated as:

$$
P_{d}^{(\text {path })}=\prod_{k=1}^{N}\left(1-P_{e}^{(h o p)}(k)\right)
$$


Provided that a packet can arrive at the sink from the source within its deadline via $M$ paths, $P_{d}^{(\text {path })}(i)$ is PDR of the $i$-th path. Duplicated packets will arrive to the sink at different times. The sink will consider only the first successfully received packet, as it will discard all latter copies. This also holds for end-to-end delay calculation. If we reorder all paths according to end-to-end delays, and denote that the packet delivered from 1st path arrives earliest and from $M$-th path arrives latest, the overall PDR from a node to the sink $P_{d}^{(\text {overall })}$ can be calculated as:

$$
\begin{array}{r}
P_{d}^{(\text {overall })}=P_{d}^{(\text {path })}(1)+\left(1-P_{d}^{(\text {path })}(1)\right) P_{d}^{(\text {path })}(2)+ \\
\\
\quad \ldots+\left(\prod_{k=1}^{M-1}\left(1-P_{d}^{(\text {path })}(k)\right)\right) P_{d}^{(\text {path })}(M)
\end{array}
$$

The partial derivatives of (4) to the PDR of $i$-th path can be written as:

$$
\frac{\partial P_{d}^{(\text {overall })}}{\partial P_{d}^{(\text {path })}(i)}=\prod_{k=1, k \neq i}^{M}\left(1-P_{d}^{(\text {path })}(k)\right), \forall i \in[1, M]
$$

Since $0 \leq P_{d}^{(\text {path })}(k)<1$, it follows that $\frac{\partial P_{d}^{(\text {overall })}}{\partial P_{d}^{\text {path }}(i)}>0$. Thus, (4) is a monotonically increasing function of PDR for each path, meaning that increase of PDR of each path implies the overall PDR increase.

As previously mentioned, the use of FEC reduces $P_{e}^{(h o p)}$ at every path, which results in higher $P_{d}^{(\text {path })}$, according to (3). Since in the proposed lightweight routing scheme all packets are FEC-coded via all paths, the PDR of any path $P_{d}^{\text {path }}(i), \forall i \in[1, M]$ is increased, compared with uncoded transmissions. Therefore, the overall PDR will be increased by the proposed FEC-coded lightweight routing scheme.

2) End-to-end delay analysis: Assuming that TDMA with slot duration $T_{t}$ is used in the MAC layer, provided a packet is transmitted at timeslot $s$ at the source and arrives at the destination at timeslot $d$, then end-to-end delay $T_{\text {delay }}$ is calculated as:

$$
T_{\text {delay }}=(d-s+1) \times T_{t}
$$

If all nodes are scheduled with timeslots according to their application deadline, without considering transmission failure, all packets are able to arrive at destinations within a deterministic time period. However, the schedulability problem is out of the scope of this paper, so we assume that all packet transmissions are schedulable.

We denote $T_{\text {delay }}(i)$ as the end-to-end delay of the $i$-th out of $M$ possible paths. If we order the paths by delay, then $T_{\text {delay }}(1)$ of the first path has the minimum value, while $T_{\text {delay }}(M)$ is the maximum end-to-end delay. In order to quantify the latency of lightweight routing transmissions, we introduce the variable $T_{\text {delay }}^{\text {weight }}$ named Weighted Average
Delay, defined as:

$$
T_{\text {delay }}^{\text {weight }}=\frac{\sum_{i=1}^{M} w_{i} T_{\text {delay }}(i)}{\sum_{i=1}^{M} w_{i}}
$$

where $w_{i}$ is the weight for delay via the $i$-th path.

We define the weight $w_{i}$ as the joint probability of a packet being unsuccessfully transmitted via the $i$-th path. Thus, $w_{i}$ can be written as:

$$
w_{i}=\prod_{k=1}^{i} P_{e}^{(p a t h)}(k)
$$

where $P_{e}^{(\text {path })}(k)$ is the PER of the $k$-th path, which can be calculated as: $P_{e}^{(\text {path })}(k)=1-P_{d}^{(\text {path })}(k)$.

This Weighted Average Delay indicates the overall latency performance of transmitting a packet from a source to the sink. Thus, the goal is to reduce the Weighted Average Delay in order to achieve lower overall latency. The partial derivative of (7) over the PER of $i$-th path is calculated as (7).

From (7), since $k \in[i, M], n \in[1, i-1]$, and we have $T_{\text {delay }}(i)<T_{\text {delay }}(j)$, if $i<j$, we can get:

$$
\frac{\partial T_{\text {delay }}^{\text {weight }}}{\partial P_{e}^{(\text {path })}(i)} \begin{cases}=0, & i=1 \\ >0, & \text { otherwise }\end{cases}
$$

Thus, (7) monotonically increases for the PER of each path, when $i \neq 1$. The conclusion is that if we can reduce PER of each path, the Weighted Average Delay can be also reduced. Since all packets in the proposed scheme are FEC-coded, the PER of all paths can be decreased. This results in the reduction of Weighted Average Delay, and, consequently, lower overall transmission latency.

\section{Simulation Setup ANd Scenarios Of INTEREST}

This section presents the simulation environment and scenarios.

\section{A. Channel model and the choice of FEC coding}

The protocol stack was designed according to the common traits of the three existing industrial communication standards, as shown in Table I. The propagation environment used in the experiments corresponds to the one observed in [4]. The channel model used is Rayleigh, with fluctuations of received SNR of around $25 \mathrm{~dB}$, a common occurrence in industrial environments.

The FEC code is chosen with respect to the timing considerations in Subsection II-A2. Namely, packets are encoded with $\mathrm{RS}(15,9)$ code, which has a similar computational complexity as FEC codes evaluated in [17].

\section{B. Simulation Scenarios}

The performance of the proposed solution is evaluated by simulation. Measurables of interest are average end-toend delay and Packet Delivery Ratio, defined earlier. Since outdated packets are discarded on-the-fly on Network layers of intermediate and terminal nodes, no outdated packets will reach the Application layer of the sink, and none of them shall 


$$
\frac{\partial T_{\text {delay }}^{\text {weight }}}{\partial P_{e}^{(\text {path })}(i)}=\frac{\sum_{m=1}^{i-1}\left(\prod_{n=1}^{m}\left(P_{e}^{(\text {path })}(n)\right) \sum_{k=i}^{M}\left(\prod_{j=1}^{k}\left(P_{e}^{(\text {path })}(j)\left(T_{\text {delay }}(k)-T_{\text {delay }}(n)\right)\right)\right)\right)}{\left(\sum_{i=1}^{M}\left(\prod_{k=1}^{i} P_{e}^{(\text {path })}(k)\right)\right)^{2} P_{e}^{(\text {path })}(i)}
$$

TABLE I

THE SIMULATION PARAMETERS

\begin{tabular}{cc}
\hline Parameter & Description \\
\hline Application layer & Contant Bit Rate application \\
Timeslot duration & $10 \mathrm{~ms}$ \\
MAC layer & TDMA \\
Physical layer & O-QPSK \\
Coding scheme & RS $(15,9)$ \\
Channel model & Rayleigh fading \\
MAC message size & 126 bytes \\
Bit rate & $250 \mathrm{kbps}$ \\
Transmit power & $10 \mathrm{dBm}$ \\
Receiver Sensitivity & $-85 \mathrm{dBm}$ \\
\hline
\end{tabular}

be considered in the average end-to-end delay calculation. The collected results refer exclusively to data packets, which carry the sensor readings. All network topologies are placed inside an area of roughly $100 \times 100 \mathrm{~m}$, with a centrally located sink. The nodes are placed randomly, with a constraint that $75 \%$ of nodes are situated one hop away from the sink, where the remaining nodes lay at a distance of two hops. Further details of the scenarios are given below.

1) Scenario I: This scenario is a comparison of the fullfledged proposed solution with its depleted version, stripped of FEC coding. The aim is to evaluate the improvements in average end-to-end delay and PDR, introduced by the FEC component of the proposed algorithm.

2) Scenario II: In this experiment, the proposed approach is compared to combinations of uncoded and coded transmissions with three well known conventional WSN routing protocols. The goal is identical to the one of Scenario I - to observe the reduction of latency and gain in reliability.

\section{Evaluation RESUlts}

This section presents the evaluation results. In the following subsections, the term "Coded lightweight" transmission scheme denotes the full-fledged solution, whereas "Uncoded lightweight" transmission scheme refers to the depleted version of the algorithm, where only the lightweight routing component is applied, while FEC coding is omitted. Qualnet discrete event simulator is used to execute the simulations.

\section{A. Scenario I}

This scenario compares the Uncoded lightweight and Coded lightweight transmission scheme. The two measurables of interest, average end-to-end delay and PDR, are observed separately. The purpose of this separation is to decouple the improvements in terms of reliability and end-to-end delay and present them individually.

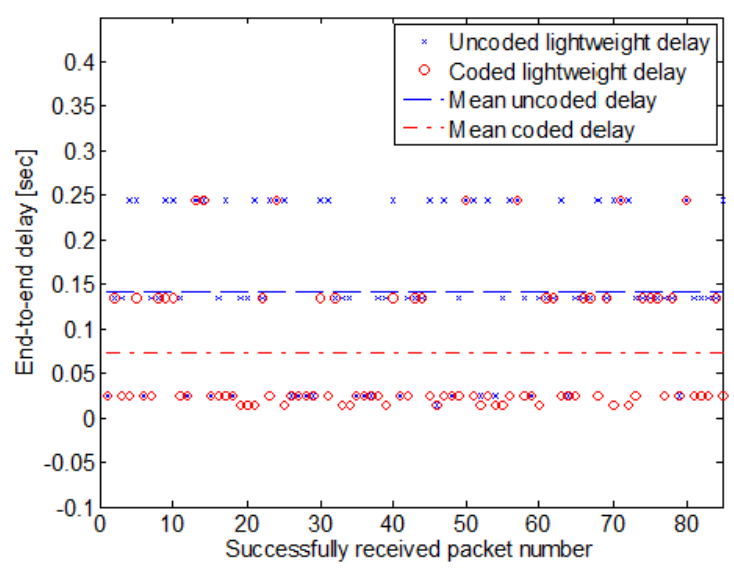

Fig. 2. End-to-end delays of particular packets in Scenario 1

1) Average end-to-end delay: The reduction of latency obtained by using FEC coding is evaluated by observing the traffic from a node located two hops away from the sink (from now on referred to as the focus node), sending at a refresh rate of $T_{c b r}=250 \mathrm{~ms}$. The network consists of four nodes in total, i.e. the focus node and three forwarders in the first tier (i.e. one hop away from the sink). The number of nodes in this scenario makes no impact on the delays from the focus node, as long as the network is schedulable. In this setting, multiple copies of the packet sent by the focus node will arrive to sink, due to existence of redundant paths. Since the MAC technique is TDMA, redundant paths from the focus node to the sink will have different delays. Figure 2 shows the obtained end-to-end delays of individual packets for uncoded and coded case, as well as their mean values. The delays of the individual coded packets are denoted by circles, while the delays of their uncoded counterparts are denoted by crosses. The set of packets considered in Figure 2 refers only to successfully delivered uncoded packets and their corresponding coded packets.

Figure 2 indicates that end-to-end-delay of the proposed solution is always less than or equal to the one of Uncoded lightweight scheme. The explanation is as follows: redundant paths occupy different timeslots and have different delays. In the ideal case, the packet copy taking the fastest path (i.e. via the earliest timeslot) would successfully arrive to the sink. This way, the contribution to average end-to-end delay would be the smallest. By applying FEC coding on the lightweight routing scheme, the probability of arrival via every path, including the 


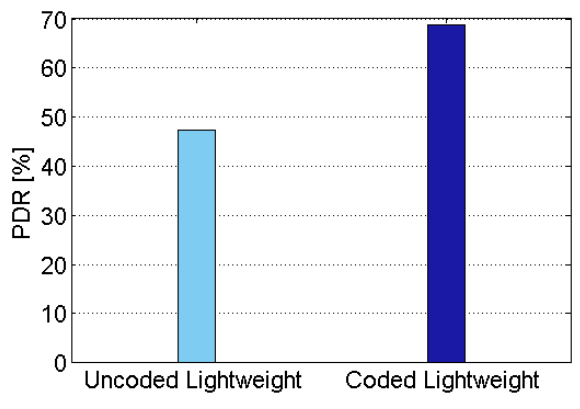

Fig. 3. PDRs of Scenario 1

fastest one, is increased, implying lower end-to-end delays. The reduction in end-to-end delay can be quantified in two ways. From Figure 2 we observe that in $49 \%$ of the cases the delay of the coded packet was smaller than the delay of its uncoded version. Another way to present the gain is via the mean values of uncoded and coded end-to-end delays shown in Figure 2, which are $142 \mathrm{~ms}$ and $74 \mathrm{~ms}$, respectively. Therefore, a $43 \%$ reduction of average end-to-end delay is achieved by the FEC component.

2) Packet Delivery Ratio: The experiments are executed on the network of 33 sensor nodes, with $25 \%$ of nodes at a two-hop distance from the centrally located sink. Sensor nodes transmit measurements at the refresh rate of $T_{c b r}=$ $500 \mathrm{~ms}$. Figure 3 compares the PDRs for uncoded lightweight transmission scheme and the full-fledged solution.

The comparison of the full-fledged and FEC-depleted version of the proposed solution shows that the FEC component of the transmission scheme contributes by an absolute PDR increase of $21 \%$, compared to uncoded transmissions. Uncoded transmissions have zero tolerance to bit errors - a single bit error irreversibly corrupts an uncoded packet. Uncoded and coded scheme have two properties in common, that affect the achieved PDRs: both schemes use redundant transmission paths, and since coding is executed on MAC layer, the 6 initial bytes of Physical layer header and preamble are unprotected, even in Coded lightweight scheme. Corruption of this part of the packet results in unconditional packet loss. On the other hand, coded transmissions have more robustness to bit errors, subject to error-correction capability of the code used, which is 3 out of 15 symbols per codeword for $\operatorname{RS}(15,9)$ code.

\section{B. Scenario II}

In this scenario, the proposed solution is compared to coded and uncoded versions of three common WSN routing protocols. The performance is observed in two aspects: average end-to-end delay (with respect to network size) and PDR.

1) Average end-to-end delay: In this experiment, the relationship between network size and average end-to-end delay is investigated. New nodes are gradually added with random placement, while maintaining the $75 \%-25 \%$ distribution of hop distance from sink. Sensor refresh rate is $T_{c b r}=1 \mathrm{sec}$.

Average end-to-end delays obtained for the proposed solution, as well as some common routing protocols are shown

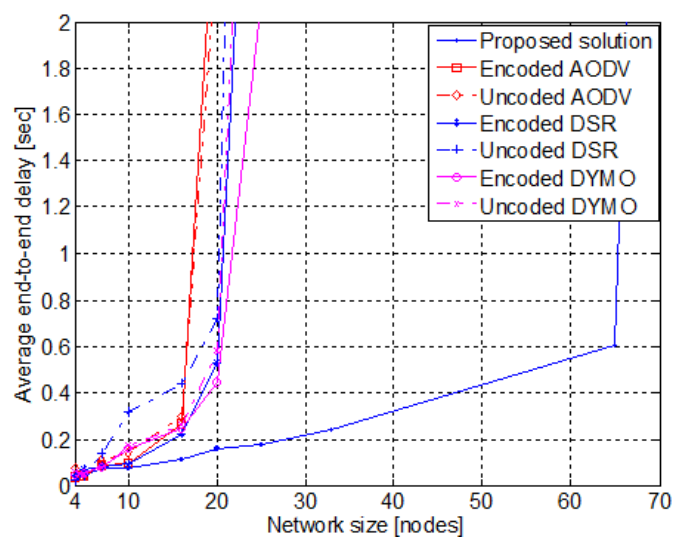

Fig. 4. Average end-to-end delays of Scenario $2\left(T_{c b r}=1 \mathrm{sec}\right)$

in Figure 4. According to the results from Figure 4, the proposed solution exhibits significantly smaller average end-toend delay than conventional routing protocols. Although coded conventional routing schemes are able to effectively reduce the average end-to-end delays, delay values still skyrocket when the network size is larger than 20 nodes. Compared with conventional routing schemes, the proposed solution shows a much less aggressive trend of end-to-end delay increase.

The medium access technique used in the experiment is TDMA, and nodes have a limited timeslot budget at their disposal. While the proposed solution encapsulates all routing information inside data packets, other routing protocols observed must allocate some timeslots for control messages, leading to the situation where data packets have to wait in output buffers for an extended period of time, because control messages have taken over their timeslots. Furthermore, the conventional routing protocols rely on establishing the transmission paths and routing tables via control message exchange, and frequent link failures will trigger frequent path recalculation processes, causing the data packets to spend more time in the buffers. This explains the huge average end-toend delays exhibited by other routing protocols. The average delay of the proposed solution skyrockets at the 65-node mark, which is the point where the proposed scheme exhausts the timeslot budget. Networks of different sizes were not built up incrementally, but with a certain step. Although there exists a certain amount of correlation between networks of subsequent sizes, adding as little as two nodes significantly affects the topology and results in a noticeable end-to-end delay increase. This explains the lack of smoothness of delay curves for the conventional routing protocols.

2) Packet Delivery Ratio: Figure 5 presents PDRs obtained for the same set of competitors as in the previous case. The network topology in this experiment is identical to the one obtained in PDR subsection of Scenario I: 33 nodes placed around a sink, with $25 \%$ of the nodes being located two hops away from the sink. The sensor refresh rate is $T_{c b r}=500 \mathrm{~ms}$.

The proposed solution achieves a higher PDR than all other transmission schemes observed, including the coded 


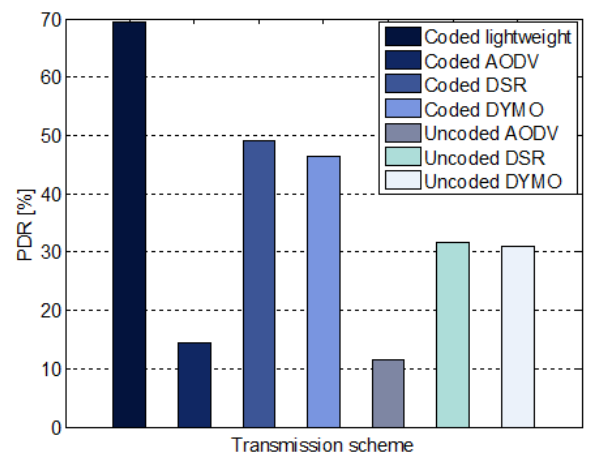

Fig. 5. PDRs of Scenario 2

conventional routing protocols. According to the results on Figure 5, the advantage in PDR is more than $20 \%$ in the worst case. The reason behind this is the existence of multiple transmission paths for nodes placed more than one hop away from the sink. In the experiment, every second-tier node has two redundant paths to the sink. The simulation parameters in this experiment are chosen so that the network is brought close to saturation, with respect to the performance of the proposed solution. Since conventional routing protocols have additional control message overhead, the network load is well above their saturation point, and this also reflects their lower PDR.

Every experiment is executed for a single value of $T_{c b r}$. It has been observed that, if the network load is below saturation, the refresh rate has no significant impact on PDR of the proposed solution, because channel coherence time in the experiments is around $5 \mathrm{~ms}$, which is two orders of magnitude less than feasible $T_{c b r}$ 's. If the network is saturated, the end-toend delays of certain packets will exceed the delivery deadline (equal to the refresh rate) and a number of packets will be discarded, resulting in lower PDR. Average end-to-end delays also do not depend on refresh rate, as long as the network is unsaturated.

\section{CONCLUSions}

In this work, a lightweight and reliable data transmission scheme for IWSN is proposed, with the aim to address some deficiencies of the existing industrial communication standards, and improve the latency and reliability of data transmission. The intervention on the MAC layer is concerned with FEC coding, while on the Network layer, a lightweight routing protocol is introduced, which significantly reduces transmission latency. The proposed solution is fully IEEE 802.15.4-compliant and may be implemented in the existing off-the-shelf hardware without violating the standard or need for intervention by the hardware vendors. Results obtained in the experiments show a substantial increase in Packet Delivery Ratio and reduced latency, compared to both coded and uncoded conventional WSN routing protocols. Both components contribute to better performance. The lightweight nature of the routing component contributes to latency reduction due to its lack of control messages and routing infrastructure.
Meanwhile, the reliability is increased because redundant paths are used to deliver packets. Apart from the obvious contribution to reliability, the coding component reduces the latency as well. Redundant paths provided by the routing component have different delays, and the use of FEC coding increases the probability that the packet will be successfully delivered via the path with the minimum delay.

Development of dynamic scheduling, data aggregation and hardware implementation are to be considered for future work.

\section{REFERENCES}

[1] Hart 7 specification, http://www.hartcomm.org/.

[2] Industrial society of automation, http://www.isa.org/.

[3] Shenyang institute of automation, http://www.industrialwireless.cn/.

[4] J. Åkerberg, M. Gidlund, F. Reichenbach, and M. Björkman, "Measurements on an industrial wireless hart network supporting profisafe: A case study," in IEEE Conference on Emerging Technologies and Factory Automation (ETFA'11), Sept. 2011.

[5] S.-Y. Ni, Y.-C. Tseng, Y.-S. Chen, and J.-P. Sheu, "The broadcast storm problem in a mobile ad hoc network," in Proceedings of the 5th annual ACM/IEEE international conference on Mobile computing and networking, ser. MobiCom '99. New York, NY, USA: ACM, 1999, pp. $151-162$.

[6] Y.-C. Tseng, S.-Y. Ni, and E.-Y. Shih, "Adaptive approaches to relieving broadcast storms in a wireless multihop mobile ad hoc network," in Distributed Computing Systems, 2001. 21st International Conference on., Apr 2001, pp. $481-488$.

[7] O. Liang, Y. Sekercioglu, and N. Mani, "A low-cost flooding algorithm for wireless sensor networks," in Wireless Communications and Networking Conference, 2007.WCNC 2007. IEEE, March 2007, pp. 3495 -3500 .

[8] H. Liu, X. Jia, P.-J. Wan, X. Liu, and F. Yao, "A distributed and efficient flooding scheme using 1-hop information in mobile ad hoc networks," Parallel and Distributed Systems, IEEE Transactions on, vol. 18, no. 5, pp. $658-671$, May 2007.

[9] C. Shanti and A. Sahoo, "Treefp: A tdma-based reliable and energy efficient flooding protocol for wsns," in World of Wireless, Mobile and Multimedia Networks (WoWMoM), 2011 IEEE International Symposium on $a$, June 2011, pp. $1-7$.

[10] F. Ferrari, M. Zimmerling, L. Thiele, and O. Saukh, "Efficient network flooding and time synchronization with glossy," in Information Processing in Sensor Networks (IPSN), 2011 10th International Conference on, April 2011, pp. 73 -84.

[11] K. Akkaya and M. Younis, "A survey on routing protocols for wireless sensor networks," Ad Hoc Networks, vol. 3, pp. 325-349, 2005.

[12] M. Hamdi, N. Essaddi, and N. Boudriga, "Energy-efficient routing in wireless sensor networks using probabilistic strategies," in Wireless Communications and Networking Conference, 2008. WCNC 2008. IEEE, 31 2008-April 3 2008, pp. 2567 -2572.

[13] L. Zhao, G. Liu, J. Chen, and Z. Zhang, "Flooding and directed diffusion routing algorithm in wireless sensor networks," in Hybrid Intelligent Systems, 2009. HIS '09. Ninth International Conference on, vol. 2, Aug. 2009, pp. $235-239$.

[14] K. Yu, F. Barac, J. Åkerberg, M. Gidlund, and M. Björkman, "A flexible error correction scheme for ieee 802.15.4-based industrial wireless sensor networks," in 21st IEEE International Symposium on Industrial Electronics (ISIE 2012), May 2012.

[15] E. Sanchez, F. Gandino, B. Montrucchio, and M. Rebaudengo, "Increasing effective radiated power in wireless sensor networks with channel coding techniques," in Electromagnetics in Advanced Applications, 2007. ICEAA 2007. International Conference on, Sept. 2007, pp. 403 -406 .

[16] F. Barac, J. Akerberg, and M. Gidlund, "A lightweight routing protocol for industrial wireless sensor and actuator networks," in IECON 2011 - 37th Annual Conference on IEEE Industrial Electronics Society, Nov. 2011, pp. $2980-2985$.

[17] K. Yu, J. Åkerberg, M. Gidlund, and M. Björkman, "Reliable and low latency transmission in industrial wireless sensor networks," in The First International Workshop on Wireless Networked Control Systems (WNCS), Sept. 2011, pp. 1-6. 